\title{
Wavelet based Multilevel Sub-Band Adaptive Thresholding for Image Denoising using Modified PSO Algorithm
}

\author{
Dinesh Kumar Gupta ${ }^{1}$, Satish Pawar ${ }^{2}$, Yogendra Kumar Jain ${ }^{3}$ \\ Research Scholar, CSE, Samrat Ashok Technological Institute, Vidisha (M.P.) ${ }^{1}$ \\ Assistant Professor, CSE, Samrat Ashok Technological Institute , Vidisha (M.P.) ${ }^{2}$ \\ Head of the Department, CSE, Samrat Ashok Technological Institute, Vidisha (M.P.) ${ }^{3}$
}

\begin{abstract}
The image de-noising is one in all the foremost studied areas within the field of image processing. There are many ways (like communication channel, imperfect sensors, interference etc.) by which the noise may affect the image. Depending upon the nature of noise and the image many techniques has been already proposed. However for any technique it is difficult to operate on different level of noises over different kind of images (like SAR images, Xray images, Ultrasound images etc.). The best possible solution for such cases is to use adaptive techniques. In this paper we are presenting a multilevel wavelet decomposition based adaptive thresholding technique which utilizes the modified Particle Swarm Optimization (PSO) algorithm to find out the optimal values for thresholds and level of decompositions for given objective function. The modification of PSO is done through random perturbation in particle velocities which induces small randomness in new particle position estimation. This randomness can effectively increase the particle search space, which ultimately provides a much better solution than the conventional PSO. Finally the proposed algorithm is validated by testing it over different kind of images corrupted by different values of noise.
\end{abstract}

Keywords: Image De-noising, Wavelet Decomposition, Adaptive Thresholding, Particle Swarm Optimization (PSO).

\section{INTRODUCTION}

The recent requirements in digital imaging, multimedia technology and social networking have promoted the research in the field of image processing. There are many image processing applications, such as compression, enhancement, face recognition, and noise removal. The noise removal is the most common and essential processing step in imaging system. Images often corrupted by noises because of machine specifications, detector specifications and surroundings. Image denoising is the process of removing noise from an image. However the image de-noising has a tradeoff which is found between noise reduction and preserve important image details [25]. To achieve a good tradeoff, a de-noising algorithm has to be capable to handle image discontinuities. There are many different kinds of image denoising methods. These methods can be classified in two categories i.e. spatial domain filtering and Transform domain filtering.

The spatial filtering works directly on image plane and manipulates the pixel value of corrupted pixel by applying various algorithms of filters [26]. The values of neighborhood pixels decide the value of processed pixel therefore it is also known as neighborhood process. Spatial filters can be further classified into non-linear and linear filters. In linear filters output values are linear function of the pixels in the original image. Linear methods are easy to analyze mathematically than the nonlinear filters. Nonlinear filters have accurate results because they are able to reduce noise levels without blurring the edges.

The image denoising method which uses wavelet transform falls into transform domain filtering. The wavelet based denoising techniques are used to remove the noise present in the signal while preserving the signal characteristic as it is, regardless of frequency content. It contains three steps: (1) a linear forward transform (2) non linear thresholding (3) linear inverse wavelet transform. The wavelet decomposition has the inherent characteristics which makes the construction of such spatially adaptive algorithms feasible. The wavelet transform arranges the essential information contained by image pixels into a number of bands called LL, LH, HL and HH. These bands can be further refined into different resolution scales. Because of such properties, the additive Gaussian noise can be effectively removed even by simple thresholding and shrinkage of the wavelet coefficients.

The noise suppression method to be proposed in this paper has been modeled as follows:

Let $f(t)$ be a original image and $g(t)$ be the image corrupted with independent and identically distributed (i. i.d.) zero mean, white Gaussian Noise $z(t)$, given in below;

$$
g(t)=f(t)+\sigma_{n} z(t)
$$

Where $z(t)$ has a normal distribution $N(0,1)$ and $\sigma_{n}$ is the noise variance.

In this paper, we further extend the adaptive thresholding approach. The main improvements are (1) sub-division of the image into smaller blocks which does not have too much pixel variations. (2) A joint level of decomposition and threshold search for each block and (3) a modified PSO algorithm for searching the optimal values for decomposition levels and thresholds. The paper is organized as follows. In Section II, similar literatures and concepts are briefly reviewed. A brief description of 
wavelet decomposition and thresholding is presented in image restoration achieves the regularization by promoting Section III; Section IV discusses the PSO and the modified a reconstruction with low complexity, wavelet PSO algorithm. The proposed technique and its practical coefficients, taking advantage of the sparsity of wavelet implementation are described in Section V. The simulation results are presented and discussed in Section VI. Finally, concluding comments are given in Section VII.

\section{LITERATURE REVIEW}

A wide range of image de-noising algorithms are based on Discrete Wavelet Transform (DWT). Donoho and Johnstone proposed a method in which additive white Gaussian noise can be effectively removed by simple thresholding of the wavelet coefficients [1]. Chipman et. al. demostrate a method which uses Bayes estimation assuming independent wavelet coefficients [2]. There are several wavelet based de-noising techniques proposed which uses image characteristics, inter-scale dependencies or intra-scale (spatial) correlations between image wavelet coefficients [3,4 and 5]. Combined inter and intra-scale dependencies in a decimated, orthonormal wavelet basis is presented in literature however it only shows minor improvement [6]. Although, in a non-decimated wavelet basis, Malfait and Roose presented technique which gives a clear advantages in terms of quantitative image quality measures as well as in visual quality of the results and combines the inter-scale and intra-scale dependencies. It uses the bi-level Markov Random Field (MRF) model to encode prior information about spatial clustering of wavelet coefficients. The inter-scale dependencies are estimated through measure of inter-scale ratios.

The statistical properties of these measures are conveyed through a conditional probability density model, and combined with the prior model to form a Bayesian network. Since the conditional model is heuristic and parameterized, which makes it very complex for practical implementation [7]. Another Bayesian network based approach is presented in literature which investigated the statistical characterization of inter scale ratios of wavelet coefficients [8]. Different local criteria for distinguishing useful coefficients from noise are evaluated; after that a joint conditional model is introduced, and finally anisotropic Markov Random Field prior model used to denoise the image. Jansen and Bultheel did not use interscale statistics, but instead used the magnitude of wavelet coefficient as its significance estimate [5 and 9].

The low complexity wavelet transform based image denoising is proposed which was inspired by simple wavelet image compression algorithm and uses the Estimation Quantization Coder. In the low complexity wavelet transform based image de-noising method, the wavelet image coefficients are assumed as zero mean Gaussian random variables with high local correlation. A Marginal prior distribution on wavelet coeffcients variances is assumed and these wavelet coefficients are estimated using an approximate Maximum a Posteriori Probability rule and an approximate Minimum Mean Squared Error estimation procedure is applied to restore the noisy wavelet image coefficients [10]. An expectationmaximization (EM) algorithm in wavelet domain for decomposition [11]. Although the idea of thresholding is easy and efficient, finding a good threshold value is not an easy task.

Donoho and Johnstone [15] proposed the universal thresholding method called VisuShrink $T_{U}=\sigma \sqrt{2 \log M}$ and is defined for one-dimensional (1-D) deterministic signal of length $M$, which results in an estimate asymptotically optimal in the minimax sense (minimizing the maximum error over all possible $\mathrm{M}$-sample signals). One other prominent threshold is the SURE threshold, derivative of minimizing Stein's unbiased risk estimate when soft-thresholding is used [16, 17]. The Sure Shrink method is a combination of the universal and the SURE threshold, with the choice being dependent on the energy of the particular sub-band [16]. The SURE threshold is data-driven, and it does not depend on M explicitly, and Sure Shrink estimates it in a sub-band adaptive manner. Furthermore, Sure Shrink provides good image de-noising performance and comes close to the true minimum MSE of the optimal soft-threshold estimator [14, 18].

Optimizing the process of wavelet thresholding image denosing by using population based metaheuristics like Particle Swarm Optimization; Genetic algorithm has been done in the recent year. Bhatuda et al proposed a PSObased approach for learning the parameters of sub-band adaptive thresholding function for image denoising [20]. Wang et al. proposed modified Chaotic Particle Swarm Optimization which used a chaotic PSO approach to optimize the wavelet threshold values [21]. Liu et al. proposed PSO shrink which explored a complete solution space for suitable threshold [22]. Du-Jin Liu et al. proposed variant of PSO, a Fast Particle Swarm Optimization for obtaining the most optimum wavelet threshold values [23].

Mukhopdhyay and Mandal proposed a denoising technique of medical images through thresholding and optimization employing a stochastic and randomized technique of Genetic Algorithm (GA). During this technique the noisy image is divided into fixed sized block and then transforms it into wavelet domain. Some necessary parameters within the 2-D discrete wavelet transform like the decomposition level and the threshold value are searched and optimized in a wide range in the proposed technique. The Bayesian shrinkage technique has been chosen for thresholding primarily based on its sub band dependency property [24].

To efficiently remove noise from image there is need to find optimized threshold value for wavelet coefficient shrinkage. The estimation of optimized threshold value will improve the performance of denoising method. In proposed work, we are estimating threshold value for wavelet coefficient by using modified PSO algorithm. The modified PSO algorithm searches the corrected threshold value and the value of decomposition level and improves PSNR of denoised image. 


\section{WAVELET THRESHOLDING AND THRESHOLD SELECTION}

In wavelet threshholding we have considered wavelet transformed matrix of given noisy image and perform thresholding on coefficient. Wavelet thresholding is a nonlinear technique in which an image or the given data is decomposed into wavelet coefficients. These wavelet transformed coefficients are then compared with a optimized threshold value, the coefficients which is smaller than the threshold are set to zero while the others are retained or modified depending on the thresholding rule. The image is then recovered from the modified coefficients, which is called Inverse Discrete Wavelet Transform (IDWT). Wavelet shrinkage denoising has the following steps:

1. Load a noisy image.

2. Compute a linear forward discrete wavelet transform of the noisy image.

3. Apply a non-linear thresholding operation on the wavelet coefficients of the noisy image.

4. Compute the linear inverse wavelet transform of the threshold wavelet coefficients.

Let the noise free input image be $i=\left\{i_{j k}, j, k \in N\right\}$, where $N$ can be integer power of 2 , has been corrupted by additive white Gaussian noise (AWGN) $n_{i j}$,i.e.

$$
g_{j k}=i_{j k}+n_{j k}, \quad j, k=1,2,3 \ldots, N .
$$

Where $\left\{g_{j k}\right\}$ is the noisy image and $\left\{n_{j k}\right\}$ is Gaussian noise. The Gaussian noise is independent and identically distributed (iid) as normal $N\left(0, \sigma^{2}\right)$ and independent of $\left\{i_{j k}\right\}$. The goal is to remove the noise, or "de-noise" $\left\{g_{j k}\right\}$, such that obtain an estimated $\left\{\hat{l}_{j k}\right\}$ of $\left\{i_{j k}\right\}$ which minimizes the mean squared error (MSE). The MSE of estimated $\left\{\hat{l}_{j k}\right\}$ is calculated as fallows

$$
\operatorname{MSE}(\hat{\imath})=\frac{1}{N^{2}} \sum_{j=1}^{N} \sum_{k=1}^{N}\left(\hat{l}_{j k}-i_{j k}\right)^{2}
$$

Let $\boldsymbol{g}=\left\{g_{j k}\right\}, \boldsymbol{i}=\left\{i_{j k}\right\}$, and $\boldsymbol{n}=\left\{n_{j k}\right\}$ show the matrix representation of the signals under consideration. Let $\boldsymbol{G}=\mathbb{w} \boldsymbol{g} I=\mathbb{w} \boldsymbol{i}$ and $N=\mathbb{w} \boldsymbol{n}$ denote the matrix of wavelet coefficients of $\boldsymbol{g}, \boldsymbol{i}, \boldsymbol{n}$ respectively where $\mathbb{w}$ is the two-dimensional dyadic orthogonal wavelet transform operator.

The readers may refer to references such as [12] [13] for details of the two-dimensional discrete wavelet transform. It is suitable to label the sub-bands of the transform as given in Fig. 1. The discrete wavelet transform decomposes the noisy image into the different frequency sub-ands $L L_{j}, L H_{k}, H L_{k}, H H_{k}$ where $k=1,2 \ldots \ldots j$ is the $\mathrm{k}$-th frequency level and $\mathrm{j}$ is the largest scale in the decomposition. A sub-band at scale $k$ has size $N / 2^{k} \times$ $N / 2^{k}$. $J$ is typically selected large enough such that $\frac{N}{2^{J}} \ll N$ and $\frac{N}{2^{J}}>1$ These all sub-bands provide different information about the image. The lowest sub-band $L L_{j}$ represents coarse information about the image. The $L H_{k}, H L_{k}, H H_{k}$ sub-bands represent the horizontal, vertical and diagonal information about the image respectively. The highest frequency sub-band is $H_{k}$. The $L L_{k}$ sub-band frequency sub-band is further decomposed in recursive manner into the sub-bands $L H_{k+1}, H L_{k+1}$, $\mathrm{HH}_{k+1}$

\begin{tabular}{|c|c|c|c|}
\hline $\mathrm{LL}_{3}$ & $\mathrm{HL}_{3}$ & & \\
\cline { 1 - 1 } $\mathrm{LH}_{3}$ & $\mathrm{HH}_{3}$ & $\mathrm{HL}_{2}$ & \\
\hline $\mathrm{LH}_{2}$ & $\mathrm{HH}_{2}$ & \\
& & \\
& & \\
& & \\
& & \\
& $\mathrm{LH}_{1}$ & \\
& & \\
& & \\
\end{tabular}

Figure.1. Sub-bands of the 2-D discrete wavelet transform on level three.

The wavelet thresholding de-noising method filters each coefficient $Y_{i j}$ from the detail subbands with a threshold function to obtain $\widehat{X}_{i j}$ (estimated de-noised coefficients).

The de-noised estimate is then $\widehat{\boldsymbol{\imath}}=\mathbb{w}^{-1} \widehat{\boldsymbol{X}}$, where $\mathbb{w}^{-1}$ is the inverse wavelet transform operator. There are two thresholding methods frequently used. The soft-threshold function (also called the shrinkage function)

$$
\eta_{T}(x)=\operatorname{sgn}(x) \cdot \operatorname{Max}(|x|-T, 0)
$$

Takes the argument and shrinks it toward zero by the threshold $T$. The other popular alternative is the hardthreshold function

$$
\psi_{T}(x)=x \cdot \mathbf{1}\{|x|>T\}
$$

Which keeps the input, if it is greater than the threshold $T$; otherwise, it is set to zero. The wavelet thresholding technique removes noise by thresholding solely the wavelet coefficients of the detail sub-bands, whereas keeping the low resolution coefficients unaffected. The soft-thresholding rule is chosen over hard-thresholding for many reasons. First, soft-thresholding has been shown to attain near-optimal mini-max rate over an oversized range of Besov spaces [14] [15]. Second, the optimal softthresholding estimator yields a smaller risk than the optimal hard-thresholding estimator. Finally, in apply the soft-thresholding technique yields a lot of visually pleasant images over hard-thresholding because hard thresholding suffers from abrupt discontinuity that causes artifacts within the restored image, particularly when the noise energy is important, whereas soft-thresholding causes the restored image over smoothing.

\section{PSO AND THE MODIFIED PSO ALGORITHM}

\section{A. Standard PSO Algorithm}

The PSO algorithm is inspired by social behavior of bird flocking or fish schooling. Eberhart and Kennedy introduced the PSO algorithm in 1995 [19]. In PSO, each particle in the population represents a possible solution of the optimization problem, which is defined by its objective/cost/fitness function. In each iteration, a new 
position of particles is calculated based on its last location and velocity.

Initially, the PSO algorithm deploys the particles randomly within the search space, and then it simply uses the objective function to estimate the fitness of each particle. Each particle maintains its position, fitness, velocity, and the best fitness value it has achieved also known as particle best or individual best solution. Finally, the PSO algorithm estimates the global best solution (particle position which gives minimum/maximum fitness value among all particles in the swarm).

The PSO algorithm can be explained in following steps:

Step 1: Let the position and velocity of particles at $k^{\text {th }}$ iteration is given by

$$
\begin{aligned}
& P^{k}=\left\{p_{1}^{k}, p_{2}^{k}, p_{3}^{k}, \ldots . . p_{N}^{k}\right\} \\
& V^{k}=\left\{v_{1}^{k}, v_{2}^{k}, v_{3}^{k}, \ldots . . v_{N}^{k}\right\}
\end{aligned}
$$

Where $N$ shows the number of particles.

Step 2: Then fitness of each particle at $k^{t h}$ iteration

$$
\begin{gathered}
F^{k}=\left\{f_{1}^{k}, f_{2}^{k}, f_{3}^{k}, \ldots \ldots \ldots \ldots f_{N}^{k}\right\} \\
f_{i}^{k}=\text { fun } n_{\text {objective }}\left(x_{i}^{k}\right)
\end{gathered}
$$

Step 3: calculate the particle best $\left(P_{\text {pest }}^{k}\right)$ and global best $\left(G_{\text {best }}^{k}\right)$ as follows:

$$
\begin{gathered}
P_{\text {best }}^{k}=\left\{p_{\text {best }, 1}^{k}, p_{\text {best }, 2}^{k}, \ldots \ldots \ldots, p_{\text {best }, N}^{k}\right\} \\
p_{\text {best }, i}^{k}=\min \left\{p_{\text {best }, i}^{0}, p_{\text {best }, i}^{1}, \ldots \ldots, p_{\text {best }, i}^{k}\right\} \\
G_{\text {best }}^{k}=\min \left\{\begin{array}{c}
f_{1}^{0}, f_{2}^{0}, \ldots, f_{N}^{0}, f_{1}^{1}, f_{2}^{1}, \ldots, f_{N}^{1}, \ldots \ldots, \\
f_{1}^{k}, f_{2}^{k}, \ldots ., f_{N}^{k} \\
i=1,2,3 \ldots, N .
\end{array}\right.
\end{gathered}
$$

Step 4: Update velocity and position of each particle

$$
\begin{aligned}
v_{i}^{k}=w^{k-1} * v_{i}^{k-1}+c_{1} \cdot r_{1}\left(p_{\text {best }, i}^{k-1}-x_{i}^{k-1}\right)+c_{2} \\
\cdot r_{2}\left(G_{\text {best }}^{k-1}-x_{i}^{k-1}\right)
\end{aligned}
$$

Where $w$ represents the inertia weight. While $r_{1}$ and $r_{2}$ are the two uniformly distributed random numbers having mean of 0 and variance of 1 .

Step 5: Update inertia and positions of each particle

$$
\begin{gathered}
w^{k}=w_{\text {start }}-\frac{\left(\left(w_{\text {start }}-w_{\text {end }}\right) * k\right)}{\text { iter }_{\text {max }}} \\
x_{i}^{k}=x_{i}^{k-1}+v_{i}^{k}
\end{gathered}
$$

The value of the inertial coefficient $w_{\text {start }}$ and $w_{\text {end }}$ are typically chosen between 0.8 and 1.2.

Step 6: Repeat the whole process till the stopping criteria meets.

\section{B. Modified PSO Algorithm}

In the modified algorithm the calculation of inertia component $w^{k}$ is modified by the following equation:

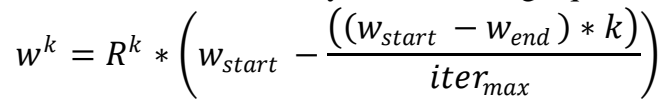

Where $R^{k}$ is calculated as

$$
R^{k}=\mu R^{k-1}\left(1-R^{k-1}\right), R^{0}=0.63, \mu=4
$$

This represents the logistic map of degree 2, and known for its complex, chaotic behaviour from very simple nonlinear dynamical equations.

The modification increases the particle search space, which ultimately provides a much better solution than the conventional PSO.

\section{PROPOSED TECHINIQUE}

For the success of wavelet based denoising, good estimation of the wavelet parameters such as wavelet function, decomposition level and threshold value is very important. These parameters are generally estimated in empirical or semiempirical manner throughout the denoising of the corrupted images. This procedure does not provide any certainty to achieve the optimal restoration result. To resolve the problem, we have proposed the addition of one randomized search algorithm to existing method. In this paper, we have proposed a wavelet based denoising technique which is based on modified PSO threshold technique. In proposed method, a randomized search algorithm i.e. modified PSO algorithm is used to search corrected threshold value and the value of decomposition level. In our proposed work, we subdivide the image into smaller block which does not have too much pixel variations.Then we use a modified PSO algorithm to search the corrected threshold value and the value of decomposition level for each block. After finding the corrected threshold value and the value of decomposition level, rearrange the blocks to form the denoised image. To illustrate our proposed technique, let the variables in sub-band adaptive thresholding image denoising problem are level of decompositions (L) and threshold (T) for each block $L=\left\{l_{1}, l_{2}, l_{3}, \ldots \ldots l_{M}\right\}, T=$ $\left\{t_{1}, t_{2}, t_{3}, \ldots \ldots t_{M}\right\}$, where $M$ is the total number of blocks. Hence the vector of a particle is group of elements corresponding to $[L, T]$. Therefore, the position of any particle at iteration $k$ can be represented as the vector $p_{i}^{k}=\left\{l_{1}^{k}, l_{2}^{k}, \ldots . ., l_{M}^{k}, t_{1}^{k}, t_{2}^{k}, \ldots \ldots t_{M}^{k}\right\} \quad$ which sets the dimension of the vector equal to $2 M$.

Now if the initial population of particle be $N$ then the complete set can be presented as:

$$
\begin{gathered}
P^{k}=\left\{p_{1}^{k}, p_{2}^{k}, p_{3}^{k}, \ldots \ldots p_{N}^{k}\right\} \\
p_{i}^{k}=\left\{l_{1}^{k}, l_{2}^{k}, \ldots \ldots, l_{M}^{k}, t_{1}^{k}, t_{2}^{k}, \ldots \ldots t_{M}^{k}\right\} \\
l_{\text {min }} \leq l_{i}^{k} \leq l_{\text {max }}, \text { for } \forall i, k \\
t_{\text {min }} \leq t_{i}^{k} \leq t_{\text {max }}, \text { for } \forall i, k
\end{gathered}
$$

Where $l_{\text {min }}, t_{\min }$ and $l_{\text {max }}, t_{\max }$ are the user defined lower and upper bounds for level of decompositions and threshold value.

Using the above mapping we can call the PSO algorithm to find the best values for $L$ and $T$ as follows:

1. Divide the image into $M$ Non-Overlapping Blocks.

2. Initialize the inertia, position and velocity of particles randomly within the defined constrains.

3. Extract the Decomposition Levels and Threshold value for each Block.

4. Perform the wavelet decomposition up to the levels extracted in Step 3.

5. Apply the thresholding in each decomposed block extracted in Step 3.

6. Take the inverse wavelet transform of each block and rearrange the block to form de-noised image.

7. Calculate the fitness of each particle using $M S E$. 
10. Update the inertia weight.

11. Check for the stopping criteria. If satisfy then exit else repeat the procedure from Step 2.

12. On exit return the particle position related to $G_{b e s t}$.

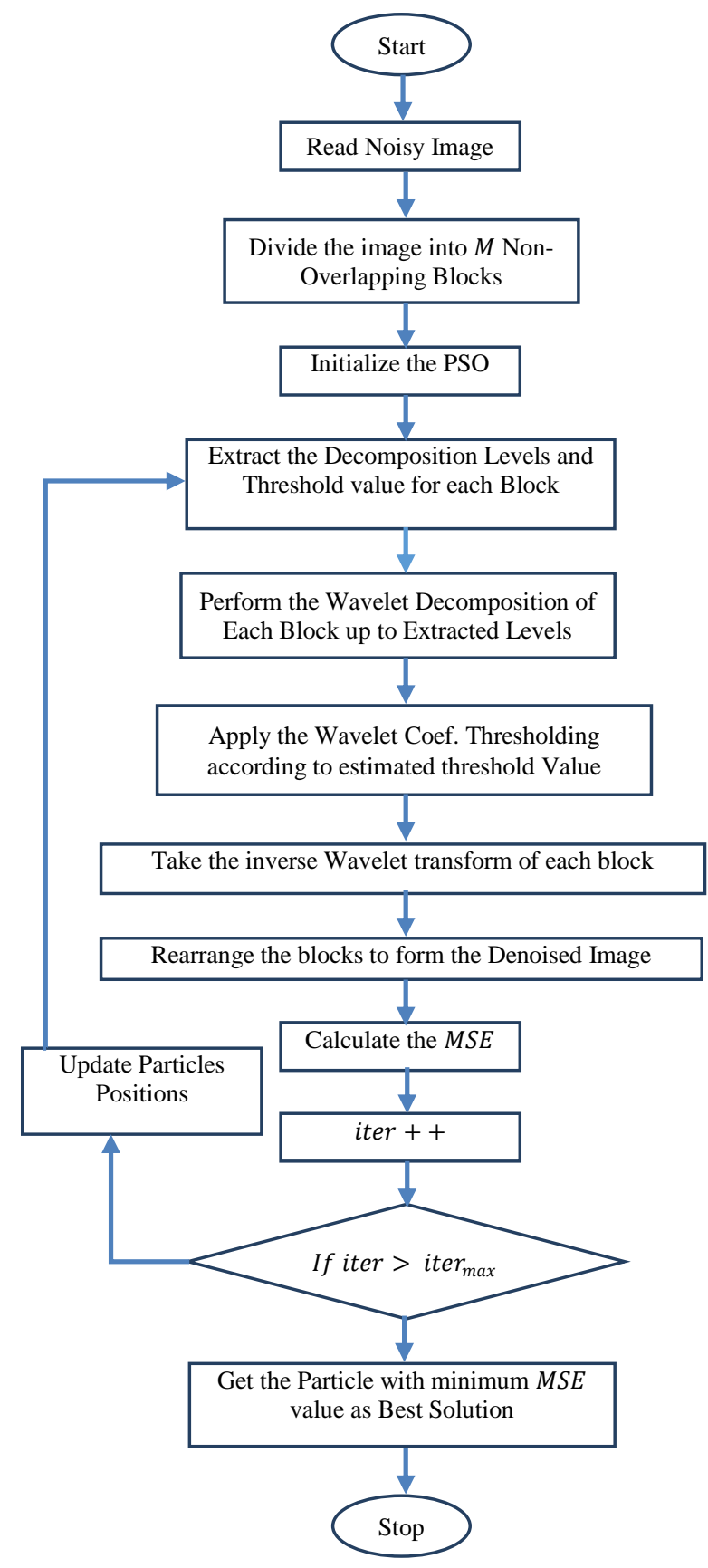

Figure 2: Flow Chart of the Proposed Algorithm.

\section{SIMULATION ENVIRONMENT AND RESULTS}

For simulation and result comparisons we have implemented our proposed technique in MATLAB (R2012b). We have applied our proposed technique on several natural grayscale images of size $256 \times 256$. For the wavelet transform of input image, we have used the Haar wavelet transform. In proposed technique, the image is subdivided into smaller blocks of size $32 \times 32$ which do not have too much pixel variations and the total number of blocks will be 64 . Since in the proposed technique for each block the decomposition level and threshold value is searched using modified PSO. Therefore total number of dimension will be 128(64 for decomposition level and 64 for threshold values). Hence the particle's search space is spread over 128 dimensions. For the effectiveness of proposed algorithm we compared with the Conventional Genetic Algorithm on different types of images like Homi, Airplane, Red fort, and Bird at different noise variance $\sigma=10,20,30,50$.

The image quality measures are very important factor in this particular problem because this term is used as fitness value in proposed algorithm. Hence if improper measure is set then the possibility of finding good results can be greatly fade. Hence in this paper we prefer the use of most common measure known as Mean Squared Error (MSE), which is defined as:

$$
M S E=\frac{1}{M N} \sum_{i=1}^{M} \sum_{j=1}^{N}(X(i, j)-Y(i, j))^{2}
$$

Where $M$ and $N$ are rows and columns in image respectively, $X$ and $Y$ represent the original and De-noised images.

Another measure known as Peak Signal to Noise Ratio $(P S N R)$ could also be taken although it is derived from $M S E$

$$
\operatorname{PSNR}(d B)=10 \log _{10}\left(\frac{255^{2}}{M S E}\right)
$$

However we prefer the $M S E$ because it minimizes with better quality and give a much larger scale resolution.

The simulation of all algorithms is performed using MATLAB. The population size $\left(N_{P}\right)$ and maximum iteration number $\left(\right.$ iter $\left._{\max }\right)$ are set as 16 and 20, respectively. Inertia weight $w_{\max }$ and $w_{\min }$ are set as 0.9 and 0.1 respectively because these values are widely accepted and verified in solving various optimization problems. The list of all values used for Modified PSO algorithm is shown in the table 1 .

Table 1: Parameter values used for Modified PSO algorithm

\begin{tabular}{|c|c|}
\hline Name of Variable & Value Assigned \\
\hline$c_{1}$ & 2 \\
\hline$c_{2}$ & 1 \\
\hline$w_{\max }$ & 0.9 \\
\hline$w_{\min }$ & 0.1 \\
\hline$\mu$ (logistic map) & 4.0 \\
\hline$k$ (logistic map) & 0.63 \\
\hline Total Particles $\left(N_{P}\right)$ & 16 \\
\hline Maximum Iterations & 20 \\
\hline
\end{tabular}

We verified our proposed denoising technique on a number of natural images. In wavelet based image denoising method, we found the optimal threshold value, the wavelet coefficient shrinkage to achieve a better denoised image. To check performance of our algorithm we have used image of Homi Bhabha which is degraded by different noise variances $\sigma=10,20,30,40$ as shown in figure 3 and it is observed that our proposed method is better than GA method.Similarly Figure 4 show the image 
of Airplane, Redfort and bird which are degraded by noise variance $\sigma=30$. To evaluate the performance of proposed algorithm, different images are considered for experiment purpose. It has been observed that proposed method provide better result as compared to wavelet based denoising method using Genetic algorithm. The over all results of test image are shown in table 2 .

\begin{tabular}{|c|c|c|c|c|c|}
\hline \multirow[t]{3}{*}{$\begin{array}{l}\text { Test } \\
\text { Image }\end{array}$} & \multirow[t]{3}{*}{$\begin{array}{c}\text { Noise } \\
\text { variance }\end{array}$} & \multicolumn{2}{|c|}{$\begin{array}{c}\text { Denoising } \\
\text { Method }\end{array}$} & \multicolumn{2}{|c|}{$\begin{array}{c}\text { Denoising } \\
\text { Method }\end{array}$} \\
\hline & & \multicolumn{2}{|c|}{ DWT with GA } & \multicolumn{2}{|c|}{$\begin{array}{l}\text { DWT with } \\
\text { MPSO }\end{array}$} \\
\hline & & MSE & PSNR & MSE & PSNR \\
\hline \multirow{4}{*}{ Homi } & 50 & 1724 & 15.76 & 473.8 & 21.37 \\
\hline & 30 & 716.9 & 19.57 & 222.2 & 24.66 \\
\hline & 20 & 324.7 & 23.01 & 136.8 & 26.76 \\
\hline & 10 & 87.92 & 28.68 & 53.49 & 30.84 \\
\hline \multirow{4}{*}{$\begin{array}{l}\text { Airpla } \\
\text { ne }\end{array}$} & 50 & 2068 & 14.97 & 506.3 & 21.09 \\
\hline & 30 & 835 & 18.91 & 241.1 & 24.30 \\
\hline & 20 & 376.7 & 22.37 & 146 & 26.48 \\
\hline & 10 & 88.25 & 28.67 & 50.44 & 31.10 \\
\hline \multirow{4}{*}{$\begin{array}{l}\text { Red } \\
\text { fort }\end{array}$} & 50 & 2131 & 14.83 & 536.1 & 20.83 \\
\hline & 30 & 841.9 & 18.87 & 249.3 & 24.16 \\
\hline & 20 & 369.8 & 22.45 & 145.2 & 26.50 \\
\hline & 10 & 88.12 & 28.67 & 51.56 & 31.00 \\
\hline \multirow{4}{*}{ Bird } & 50 & 2068 & 14.99 & 371 & 22.43 \\
\hline & 30 & 836.3 & 18.91 & 98.93 & 28.17 \\
\hline & 20 & 367.4 & 22.47 & 64.96 & 30.00 \\
\hline & 10 & 88.28 & 28.67 & 29.72 & 33.39 \\
\hline \multirow{4}{*}{ Lena } & 50 & 2143 & 14.82 & 530.19 & 20.88 \\
\hline & 30 & 859.05 & 18.79 & 268.96 & 23.83 \\
\hline & 20 & 385.6 & 22.26 & 164.36 & 25.97 \\
\hline & 10 & 94.32 & 28.38 & 86.70 & 28.75 \\
\hline \multirow{4}{*}{$\begin{array}{l}\text { Camer } \\
\text { aman }\end{array}$} & 50 & 2062.2 & 14.98 & 539.90 & 20.80 \\
\hline & 30 & 761.82 & 19.31 & 251.16 & 24.12 \\
\hline & 20 & 363.44 & 22.52 & 143.40 & 26.56 \\
\hline & 10 & 93.55 & 28.42 & 53.41 & 30.85 \\
\hline \multirow{4}{*}{$\begin{array}{l}\text { Barbar } \\
\text { a }\end{array}$} & 50 & 2139.3 & 14.82 & 833.03 & 18.92 \\
\hline & 30 & 856.94 & 18.80 & 336.57 & 22.86 \\
\hline & 20 & 390.28 & 22.21 & 340.44 & 22.81 \\
\hline & 10 & 93.06 & 28.44 & 83.50 & 28.91 \\
\hline \multirow{4}{*}{ Paper } & 50 & 2181.1 & 14.74 & 833.77 & 18.92 \\
\hline & 30 & 833.73 & 18.92 & 246.80 & 24.20 \\
\hline & 20 & 330.14 & 22.33 & 133.37 & 26.88 \\
\hline & 10 & 93.75 & 28.41 & 87.95 & 28.68 \\
\hline \multirow{4}{*}{$\begin{array}{l}\text { Goldhi } \\
\text { ll }\end{array}$} & 50 & 2149.8 & 14.80 & 1049.4 & 17.92 \\
\hline & 30 & 839.03 & 18.89 & 295.68 & 23.42 \\
\hline & 20 & 383.39 & 22.29 & 159.06 & 26.11 \\
\hline & 10 & 84.13 & 28.88 & 55.45 & 30.69 \\
\hline \multirow{4}{*}{ Boat } & 50 & 2236.3 & 14.63 & 865.29 & 18.75 \\
\hline & 30 & 815.65 & 19.06 & 371.79 & 22.42 \\
\hline & 20 & 364.21 & 22.51 & 155.10 & 26.22 \\
\hline & 10 & 93.28 & 28.43 & 64.89 & 30.00 \\
\hline
\end{tabular}

Table 2: Experiment Results for different test Images corrupt by noise of different variance

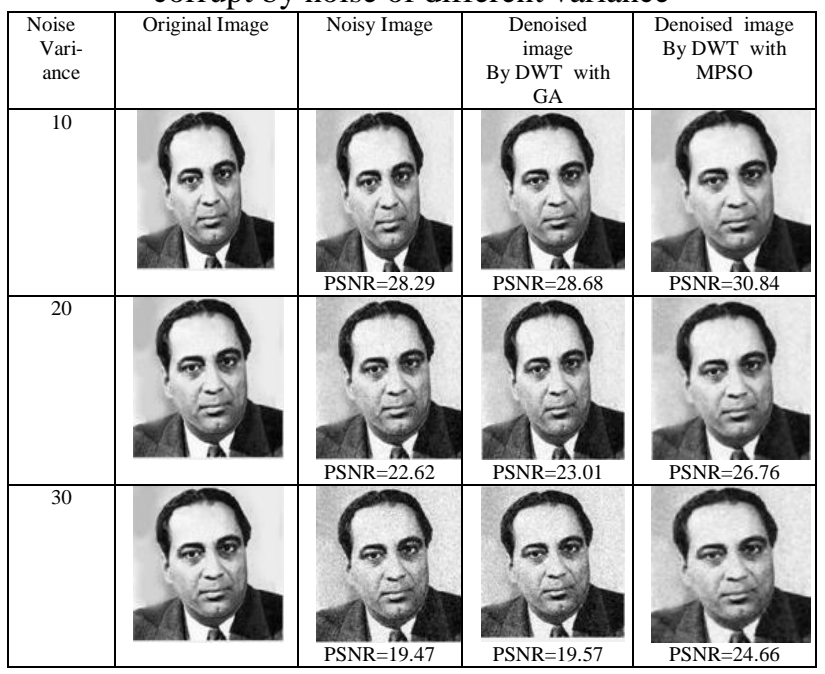

Figure3: Comparisons of PSNR value of denoised image of Homi Bhabha by DWT with GA and MPSO

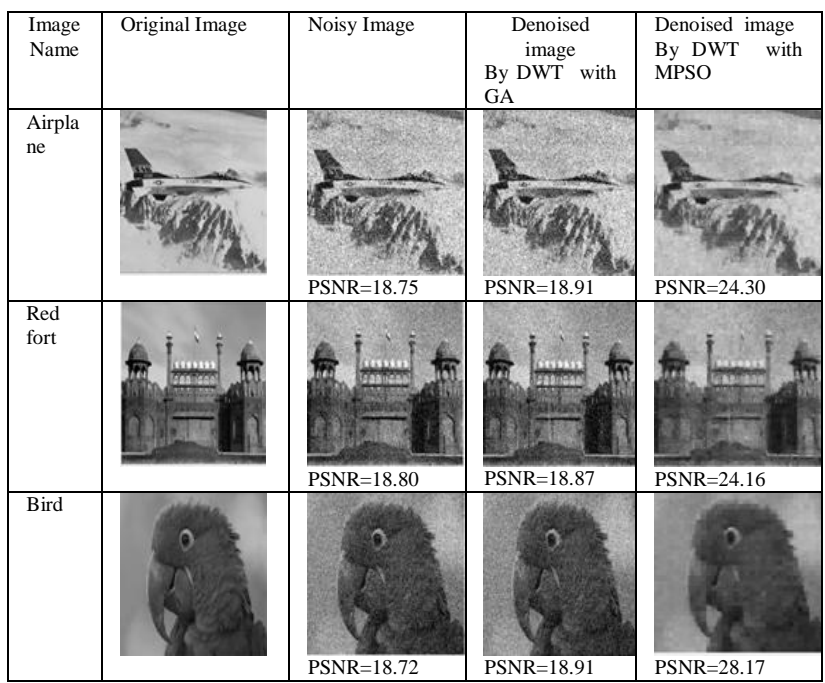

Figure4: Comparisons of PSNR value of denoised image of Airplane, Redfort, and Bird by DWT with GA and MPSO for noise variance $(\sigma=30)$

\section{CONCLUSION}

This paper proposed a Modified PSO based sub-band multilevel adaptive thresholding technique for image denoising. The proposed method exploits strength of Wavelet domains transform and capability of MPSO to obtain the good filteration results. The idea of searching the threshold value in different decomposition component is based on the fact that property of wavelet coefficients depends upon the nature of image. Implementation results show that the impact of noise is greatly reduced as compared to existing method. Proposed method is also tested for most of the common images and the simulation result shown that proposed method is more efficient and robust as compared to previous method.

\section{REFERENCES}

[1] D. L. Donoho and I. M. Johnstone, "Adapting to unknown smoothness via wavelet shrinkage," J. Amer. Stat. Assoc., vol. 90, pp. 1200-1224, 1995. 
[2] H.A. Chipman, E.D. Kolaczyk, and R.E. McCulloch, "Adaptive Bayesian Wavelet Shrinkage," J. of the Amer. Statist. Assoc., vol. 92, pp. 1413-1421, 1997.

[3] A. Cohen and J. Kova cevi'c, "Wavelets: The Mathematical Background," Proc. of the IEEE, vol. 84, pp. 514-522, 1996.

[4] R.R. Coifman and D.L. Donoho, "Translation-invariant denoising," in Wavelets and Statistics, A. Antoniadis and G. Oppenheim, editors, pp. 125-150, New York: Springer Verlag, 1995.

[5] M. Jansen and A. Bultheel, "Geometrical Priors for Noise-free Wavelet Coefficient Configurations in Image De-noising," in Bayesian inference in wavelet based models, P. M"uller and B. Vidakovi'c, editors, Springer Verlag, pp. 223-242, 1999.

[6] J. Liu and P. Moulin, "Analysis of interscale and intrascale dependencies between image wavelet coefficients", in Proc. Int. Conf. on Image Proc., (ICIP), Vancouver, Canada, Sep. 2000.

[7] M. Malfait and D. Roose, "Wavelet-Based Image Denoising Us-ing a Markov Random Field a Priori Model,” IEEE, Trans. On Image Proc., vol. 6, pp. 549-565, April 1997.

[8] Aleksandra Pizurica, Wilfried Philips, Ignace Lemahieu, and Marc Acheroy "A Joint Inter- and Intrascale Statistical Model for Bayesian Wavelet Based Image Denoising", IEEE, Trans. On Image Proc., vol. 11, no. 5, pp 545-557, May 2002.

[9] M. Jansen and A. Bultheel, "Empirical Bayes approach to improve wavelet thresholding for image noise reduction," J. of the Amer. Statist. Assoc., vol. 96, no. 454, pp. 629-639, 2001.

[10] M. Kivanc Mihcak, Igor Kozintsev, KannanRamchandran and Pierre Moulin "Low-complexity image denoising based on statistical modeling of wavelet coefficients", Signal Processing Letters, IEEE (Volume:6, Issue: 12 ) DEC. 1999.

[11] Mário A. T. Figueiredo, and Robert D. Nowak "An EM Algorithm for Wavelet-Based Image Restoration”, IEEE, Trans. On Image Proc., vol. 12, no. 8, Aug 2003.

[12] S. Mallat, "A theory for multiresolution signal decomposition: The wavelet representation," IEEE Trans. Pattern Anal. Machine Intell., vol. 11, pp. 674-693, July 1989.

[13] M. Vetterli and J. Kova cevic', Wavelets and Subband Coding. Englewood Cliffs, NJ: Prentice-Hall, 1995.

[14] ] R. A. DeVore and B. J. Lucier, "Fast wavelet techniques for nearoptimal image processing," in IEEE Military Communications Conf. Rec. San Diego, vol. 3, pp. 1129-1135, Oct 1992.

[15] ] D. L. Donoho and I. M. Johnstone, "Ideal spatial adaptation via wavelet shrinkage," Biometrika, vol. 81, pp. 425-455, 1994.

[16] David L.Donoho and Iain M.Johnstone "Adapting to unknown smoothness via wavelet shrinkage," Journal of the American Statistical Assoc., vol. 90, no. 432, pp. 1200-1224, Dec 1995.

[17] C. M. Stein, "Estimation of the mean of a multivariate normal distribution," Ann. Statist., vol. 9, no. 6, pp. 1135-1151, 1981

[18] A. Chambolle, R. A. DeVore, N. Lee, and B. J. Lucier, "Nonlinear wavelet image processing: Variational problems, compression, and noise removal through wavelet shrinkage," IEEE Trans. Image Processing, vol. 7, pp. 319-335, 1998.

[19] J. Kennedy, R. Eberhart, Particle Swarm Optimization, Proceedings of IEEE International Conference on Neural Networks. IV., 1995, pp.1942-1948.

[20] Bhutada, G. G., R. S. Anand, and S. C. Saxena. "PSObased learning of sub-band adaptive thresholding function for image denoising." Signal, Image and Video Processing 6.1 (2012): 1-7.

[21] Wang, Xuejie, Yi Liu, and Yanjun Li. "A Novel Wavelet Threshold Optimization via PSO for Image Denoising." In Computational Intelligence and Design(ISCID), 2011 Fourth International Symposium on, Vol.1, pp. 352-355. IEEE, 2011.

[22] Chan-Cheng Liu, Tsung-Ying Sun, Shang-Jeng Tsai, Yu-Hsiang Yu, Sheng-Ta Hsieh, Heuristic wavelet shrinkage for denoising, Applied Soft Computing, Volume 11, Issue 1, January 2011, Pages 256-264.

[23] Du-jin Liu, Siming Li, Shuxia Sun, Zhaoyu Ding. “Application of Fast Particle Swarm Optimization Algorithm in Image Denoising", Recent Advances in Computer Science and Information Engineering.

[24] Somnath Mukhopdhyay and J.K. Mandal "Wavelet based Denoising of Medical Image using Sub-band Adaptive Thresholding through Genetic Algorithm" ELSEVIER, vol.10 pp.680-689, 2013.

[25] Sachin D Ruikar, Dharmpal D Doye "Wavelet Based Image Denoising Technique", (IJACSA) International Journal of
Advanced Computer Science and Applications, Vol. 2, No.3, March 2011.

[26] Raghad Jawad Ahmed "Image Enhancement And Noise Removal By Using New Spatial Filters" U.P.b. Sci. Bull., Series C, Vol. 73, Iss. 1, 2011. 